\title{
A retrospective review of fatal electrocution cases at Tygerberg Forensic Pathology Services, Cape Town, South Africa, over the 5-year period 1 January 2008 - 31 December 2012
}

\author{
S von Caues, ${ }^{1}$ MB ChB; C I Herbst, ${ }^{2}$ MB ChB, Dip For Med (SA) Path, FC For Path (SA), MMed (For Path); \\ S A Wadee, ${ }^{2}$ BSc, MB ChB, MMed (For Path), FC For Path \\ ${ }^{1}$ Tonga Hospital, Mpumalanga, South Africa \\ ${ }^{2}$ Division of Forensic Medicine, Department of Pathology, Faculty of Medicine and Health Sciences, Stellenbosch University, Cape Town, \\ South Africa
}

Corresponding author: $S$ von Caues (shauneenvc@gmail.com)

\begin{abstract}
Background. Electrocution as a cause of death has been discussed extensively in the international literature. However, research on this topic in South Africa (SA) is scarce.

Objectives. To address the need for further research in this field and emphasise the necessity for preventive measures by determining the demographic and pathological profile of fatal electrocution cases seen in the Tygerberg Forensic Pathology Services, Western Cape Province, SA. Methods. The study was a retrospective and descriptive case series of all the cases of death secondary to electrocution referred to the study facility from 1 January 2008 to 31 December 2012.

Results. A total of 39 cases were included. Ten victims (25.6\%) were aged $<13$ years. The geographical area most affected by electrocution deaths was the informal settlement Khayelitsha ( $56.4 \%$ of cases). The primary injuries described were mainly burn wounds ( 34 cases, $87.2 \%)$ and abrasions (4 cases, 10.3\%). Most injuries were to the upper limbs. Unfortunately, the results pertaining specifically to the pathology of electrical burn wounds were inconclusive.

Conclusions. In view of discrepancies found in the reporting of electrical burn wounds, a standardised system for classifying these wounds is suggested. Although electrocution-related mortality is not a leading cause of death in high-prevalence areas, awareness should be raised.

S Afr Med J 2018;108(12):1042-1045. DOI:10.7196/SAMJ.2018.v108i12.13131
\end{abstract}

Electrocution-related mortality is a common topic in the international literature. Interestingly, Gupta et al. ${ }^{[1]}$ noticed that there is a distinguishable difference globally between the pattern of electrocution deaths in the Western world and that in low-income countries such as India, Iran and Turkey. ${ }^{[1-3]}$ Electrocution is an unusual occurrence in high- and middle-income countries, presumably owing to better safety precautions and increased awareness of the dangers associated with electricity. Suicidal electrocutions are more common in the Western world, while accidental fatalities are more prevalent in lowincome countries. ${ }^{[1]} \mathrm{A}$ study by Lindström et al. ${ }^{[4]}$ in Sweden compared the demographic profiles of 'occupational deaths' with 'leisure-time deaths'. All the occupational electrocution fatalities were among men. This finding may be due to the stereotype that electrical work is reserved for men. Gender-related exposure to electricity therefore explains why males are the victims in the majority of electrocutionrelated deaths, both in the workplace and at home. Lindström et $a l .{ }^{[4]}$ specifically evaluated the possible risk associated with alcohol in electrocution fatalities. Alcohol was detected in the blood in one-fifth of the victims, but the prevalence of alcohol involvement in the leisure-time deaths (42\%) was much higher than that in the occupation-related cases (5\%). Another significant finding discussed by these authors was the remarkable decrease in electrocution-related deaths over the course of the 25-year study period, highlighting the importance and efficacy of prevention strategies.

Sheikhazadi et al. ${ }^{[2]}$ conducted a study in Tehran, Iran, and noted that the most probable cause of occupation-related injuries was poor safety training. ${ }^{[2]}$ In this study, the majority of fatalities were workrelated accidents that occurred in summer. The researchers also noted a predominantly male victim profile, with a mean age of $\sim 29$ years. In $66.3 \%$ of the cases, the upper extremities were considered to be the primary contact site. A study in India described the majority of cases as sudden accidents, mostly occurring within households. ${ }^{[5]}$ This contrasts with the findings in Sweden. Gupta et al. ${ }^{[1]}$ suggested various reasons for the higher incidence of domestic electrocutions in India compared with other countries. These include a high level of illiteracy, lack of awareness about electrical hazards, and poor maintenance of electrical equipment in a low-income country. The majority of these cases occurred in the rainy season. In terms of the pattern of injuries, the profile described in India was very similar to that reported by other international authors. ${ }^{[5]}$ Entry marks were only seen in $47.1 \%$ of the electrocution fatalities studied by Gupta et al.$^{[1]}$ Non-electrocution injuries were also noted in a few cases, mainly due to secondary causes such as falls from a height. ${ }^{[1]} \mathrm{A}$ similar demographic and pathological profile was seen in a retrospective descriptive study conducted in Ankara, Turkey ${ }^{[3]}$ Entry lesions were found on the upper limbs in $74 \%$ of electrocution fatalities. Other traumatic lesions due to secondary injuries were detected in 30 cases (30\%).

In South Africa (SA), there is a paucity of data and published research on the pathological and demographic profile of deaths from electrocution. Regrettably this cause of death has not been adequately explored in SA or on the African continent. Blumenthal ${ }^{[6]}$ was the first to conduct an SA study, focusing on the burden of electrocution 
in Gauteng Province. He concluded that electrocution fatalities 'constitute a serious problem' in SA. Furthermore, he emphasised the need for further research in this field in order to address the necessity for both preventive measures and more thorough medicolegal investigation into electricity-related deaths in southern Africa.

\section{Objectives}

To describe the demographics and pattern of injuries in fatal electrocution cases seen at the Tygerberg Forensic Pathology Services (FPS) over a 5-year period. This retrospective description of all electrocution cases from 1 January 2008 to 31 December 2012 should increase insight into the extent of electrocution fatalities in the Eastern Metropole of the City of Cape Town.

\section{Methods}

The study was a retrospective and descriptive case series. The study population included all cases with a history of electrocution/'electric shock' referred for medicolegal autopsy at the Tygerberg FPS from 1 January 2008 to 31 December 2012. The FPS investigates all unnatural deaths in the Eastern Metropole of the City of Cape Town.

For every electrocution case, the final autopsy/postmortem examination report, the South African Police Service report that accompanies the body to the mortuary, the FPS form completed by the clinician in hospitalised cases reporting all clinical findings and medical and/or surgical treatment, additional questionnaires completed by forensic pathology officers when interviewing the family of the deceased, hospital records, and any additional statements added to the postmortem examination dossier were reviewed.

During the 5-year study period, 40 cases identified as having a history of fatal electrocution were seen in the facility. One case was later excluded from the study because the exact cause of death was uncertain and could not be confirmed by the available history and autopsy findings. A total of 39 cases were therefore included.

Three case files were incomplete in respect of the reports listed above, but the available history and postmortem findings confirmed electrocution to be the final cause of death and it was therefore decided to include these cases, since significant demographic and pathological findings could be obtained from the available data.

Permission to conduct this study and utilise the postmortem reports for data collection was approved by the Director of the Western Cape Forensic Pathology Services. Ethics approval was granted by the Health Research Ethics Committee, Faculty of Medicine and Health Sciences, Stellenbosch University (ref. no. S14/10/254).

Statistical analysis was performed in conjunction with the Department of Biostatistics, Stellenbosch University. The data collection was done in the Division of Forensic Medicine, Department of Pathology, Faculty of Medicine and Health Sciences, Stellenbosch University. All entered data were stored on a password-protected computer and the information used in the study was anonymised by assigning study numbers to individual cases. Confidentiality was ensured throughout the data collection process.

\section{Results}

A total of 39 cases were included in the study, $0.5 \%$ of the total of 8351 unnatural deaths investigated at the study facility between 2008 and 2012. Thirty-two victims were male $(82.1 \%)$ and 7 were female (17.9\%). Three victims (7.7\%) were unidentified at the time of autopsy. With regard to age distribution (Fig. 1), the age group with the highest prevalence was $21-35$ years $(n=15,38.5 \%)$. The majority of the victims were black African $(n=27,69.2 \%), 9$ were of mixed

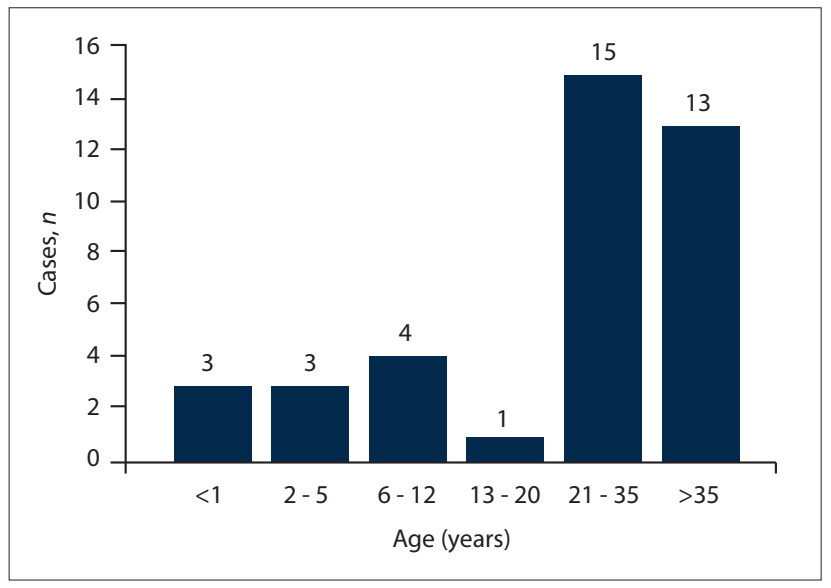

Fig. 1. Age distribution of victims of fatal electrocution.

ethnic origin (23.1\%), 2 were white $(5.1 \%)$, and 1 victim's race was unknown owing to incomplete postmortem reports.

With regard to the time and place in which the incidents occurred, 14 cases $(35.9 \%)$ occurred in summer (December - February), 11 (28.2\%) in autumn (March - May), 10 (26.6\%) in winter (June August) and $4(10.3 \%)$ in spring (September - November). Sixteen incidents (41.0\%) occurred at night (18h00 - 06h00) and 23 (59.0\%) during the day $(06 \mathrm{~h} 00$ - 18h00). The geographical area most affected by electrocution deaths in this study was Khayelitsha, with $56.4 \%$ of the cases (Fig. 2). Khayelitsha is one of the largest informal settlements in Cape Town and has a population of 391 749 people in an area of $38.71 \mathrm{~km}^{2}$, resulting in a population density of 10120 persons $/ \mathrm{km}^{2} ; 18.8 \%$ of this population have no income at all. ${ }^{[7]}$

The locations of the deaths, as described in the available case reports, are presented in Fig. 3. Of the victims who were brought to a healthcare facility, 2 were declared dead on arrival, 2 survived the initial electric shock (electrocution) and died in the healthcare facility, and the status of the remaining 2 hospitalised victims was unknown. Four deaths (10.3\%) occurred at a train station or involved 'live wires' next to a railway line.

Manner of death, i.e. the circumstances leading to a death, may be natural, accidental, homicidal, suicidal, iatrogenic or undetermined. ${ }^{[8]}$ It is important to note that the manner of death is not determined by the forensic pathologist. The breakdown of the presumed manner of deaths in this study was as follows: 35 accidental, 2 suicidal, and 2 unknown owing to missing or incomplete police reports. Only 2 cases were described as occupation-related incidents, although 5 other cases were associated with the victims working on electrical cables and wiring.

Five deaths $(12.8 \%)$ were due to an electric shock from an alleged illegal electrical connection. Another 3 cases (7.7\%) resulted from the victim's apparent attempts at cable theft. Hand tools such as metal pliers and screwdrivers were found at the scene in all 3 of these cases.

Low voltage refers to an electrical source with a voltage of $<1000 \mathrm{~V}$ and pertains mostly to the electrical current supplying most homes. Thirty-one cases in this study (79.5\%) were low-voltage electrocution deaths. Eight cases (20.5\%) were high-voltage electrocution deaths, meaning that an electrical source of $>1000 \mathrm{~V}$ caused the death. In this study the 8 high-voltage deaths included deaths associated with cable theft and a death due to the victim establishing an illegal connection. The remaining high-voltage deaths occurred at railway stations. The above distinction was made based on the history provided in each individual case. 


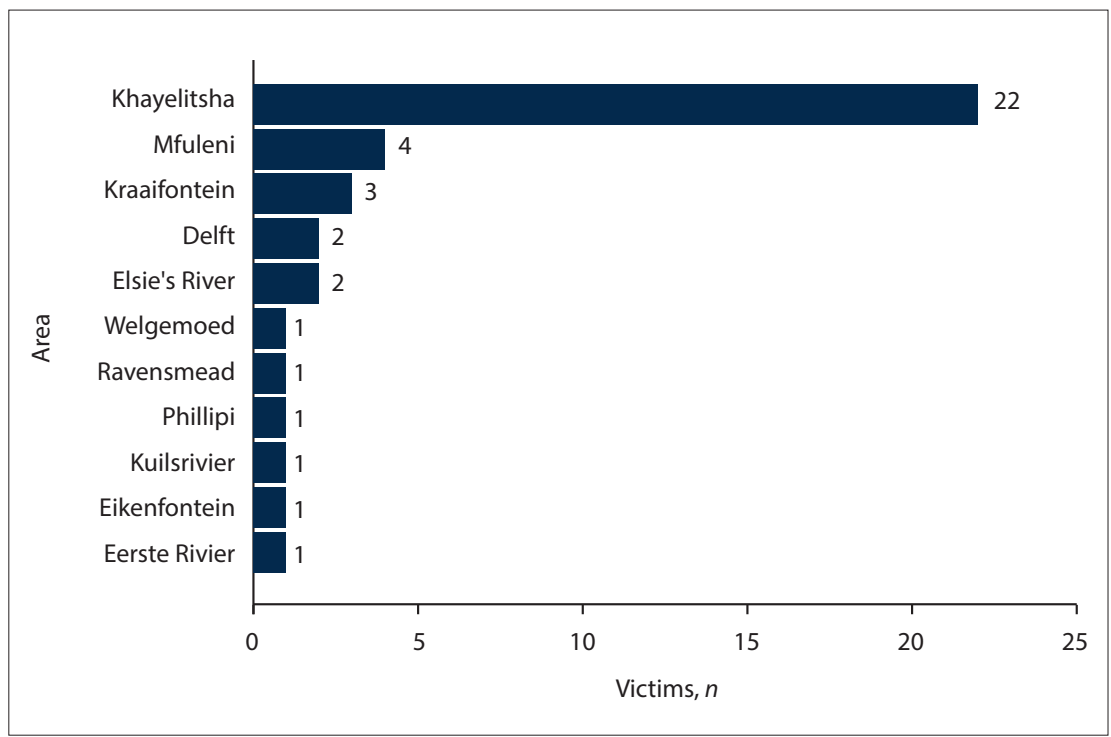

Fig. 2. Distribution of victims of fatal electrocution according to area.

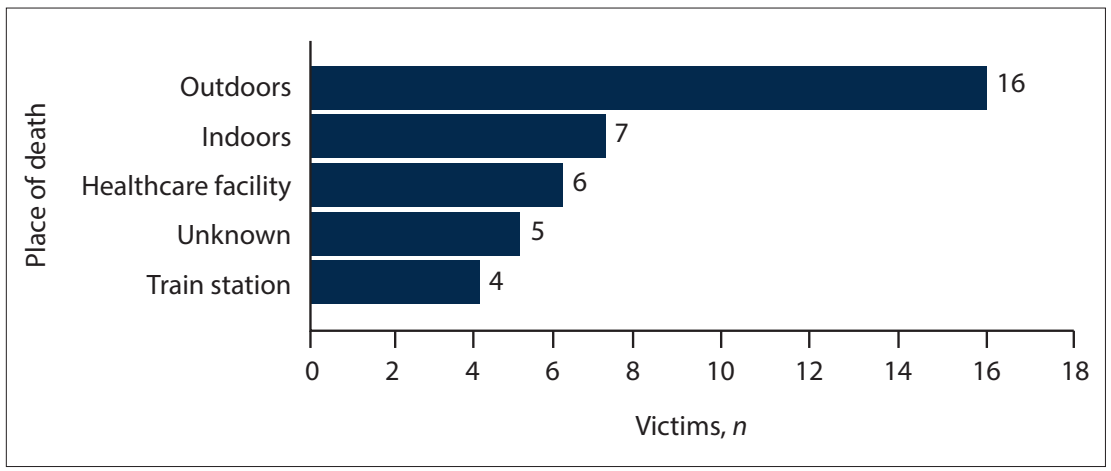

Fig. 3. Distribution of victims of fatal electrocution according to place of death.

The primary injuries described at autopsy were mainly burn wounds ( $n=34,87.2 \%)$ and abrasions $(n=4,10.3 \%)$. In 1 case, no sign of injury was found at autopsy and electrocution was finalised as the cause of death based on the presenting history.

Injuries to the upper limbs were more frequent than those to the lower limbs. Sixty-four percent of the cases $(n=25)$ had injuries to the upper limb; 13 of these cases involved only the right upper limb, while 3 cases involved both upper limbs. Unfortunately based on the available data it was not possible to distinguish entry wounds from exit wounds.

Table 1 sets out the different types of burn wounds found at autopsy. Many victims had more than one type of burn wound.

Non-electrical injuries were found in 30 cases and included abrasions, contusions, small lacerations and iatrogenic injuries due to medical intervention. Only 2 cases had signs in keeping with secondary injury as a result of electrocution. These were nonelectrical injuries suggesting a fatal fall and tongue biting, respectively.
Congestion of the internal organs, which is a common nonspecific postmortem finding, was noted in the majority of cases $(n=36,92.1 \%)$. Nine of the 36 cases showed congestion of a single organ, whereas in 27 cases congestion of multiple organs was confirmed.

Blood alcohol levels were requested in 27 cases, but only 3 tested positive for the presence of alcohol in the blood, levels ranging from $0.01 \mathrm{~g} / 100 \mathrm{~mL}$ to $0.15 \mathrm{~g} / 100 \mathrm{~mL}$.

Histological examination of the burn wounds was performed in 17 cases. The histological findings in 2 of these cases were unremarkable. The descriptions of the burn wounds in the remaining 15 cases are listed in Table 2. These histological descriptions are in keeping with the terminology used to describe both electrical and thermal burn wounds. ${ }^{[9]}$

\section{Discussion}

Despite study limitations such as a small study population and incomplete case reports, valuable conclusions could be drawn from this retrospective review.
Although electrocution is an unusual cause of death in the Eastern Metropole of Cape Town, mortality and morbidity as a result of electric shock can mostly be prevented. As reported in most of the available literature, ${ }^{[1-4,6]}$ we found that young adult men (21 - 35 years) were most exposed to electricity and therefore at a higher risk for electrocution. This finding also supports Lindström et al's observation ${ }^{[4]}$ regarding gender-related exposure to electricity.

A concerning finding was the number of young infants and children who were victims of elecrocution. In Blumenthal's study, ${ }^{[6]}$ $19.4 \%$ of the victims were aged $<25$ years. Likewise, $15 \%$ of cases in the study by Akcan et al. ${ }^{[3]}$ were between 11 and 20 years of age, with only $7 \%$ of the study population aged $<10$ years. However, compared with these studies, the burden of electrocution was higher in the younger age groups in our study; 10 victims $(25.6 \%)$ were aged $<13$ years and 6 were aged $<5$ years $(15.4 \%$ of the study population). The youngest victim in this study was only 8 months old, and was electrocuted when she touched an open wire while playing in her home.

Thirty-six percent of electrocution fatalities occurred during summer (December, January and February), which is in keeping with the findings of other authors. ${ }^{[3,6,10]}$ Some studies have noted that seasonal variation coincides with the particular area's rainy season or humidity, proposing an increased risk of fatal electrocution as a result of decreased skin resistance. ${ }^{[1,5,11]}$ Both SA studies found that most electrocution fatalities occurred during the summer ${ }^{[6]}$ despite the fact that the Gauteng climate differs significantly from that of the Western Cape Province - the rainy season in Gauteng occurs during the summer, but in the Western Cape, where this study was conducted, it occurs in winter. The theory regarding the effect of rain or humidity on the risk profile of electrocution fatalities therefore remains questionable.

The need for safety measures in informal settlements such as Khayelitsha is highlighted in this study. Illegal wiring was definitely associated with $12.8 \%$ of the deaths (5 cases), and 4 of these incidents occurred in Khayelitsha. In addition, according to the available case reports, a definite association between electrocution and illegal wiring could neither be confirmed nor rejected in 10 cases (25.6\%). Sixty percent of these 'unknown' cases also occurred in Khayelitsha. It could be argued that the few known cases associated with illegal wiring are not a true reflection of 


\begin{tabular}{ll} 
Table 1. Types of burn wounds $(\mathbf{N}=\mathbf{3 4})^{*}$ \\
\hline Type of wound & $\boldsymbol{n}(\%)$ \\
\hline Crateriform & $16(47.1)$ \\
Blistering & $15(44.1)$ \\
Parchment-like lesion & $11(32.4)$ \\
Crocodile skin & $2(5.9)$ \\
Charring & $2(5.9)$ \\
${ }^{*}$ Many victims had more than one type of burn wound. &
\end{tabular}

Table 2. Histological descriptions of burn wounds $(N=15)^{*}$

\begin{tabular}{ll}
\hline Description & $\boldsymbol{n}(\%)$ \\
\hline Blistering & $10(66.7)$ \\
Streaming & $10(66.7)$ \\
Necrosis & $6(40.0)$ \\
Palisading & $4(26.7)$ \\
Elongation & $3(20.0)$ \\
Hypereosinophilia & $2(13.3)$ \\
Pyknosis & $2(13.3)$ \\
${ }^{*}$ More than one description pertained in some cases. &
\end{tabular}

the effect illegal wiring may have on electrocution deaths. The proportion of deaths due to illegal electrical connections may in fact be much greater.

With regard to occupational deaths in this case series, only 2 deaths (5.1\%) were reported to be work related. However, an additional 5 deaths were known to be have resulted from victims attempting to fix electrical equipment. These findings suggest two important target areas of concern: poor safety training and implementation of regulations in the workplace, and attempts by unskilled individuals to repair electrical appliances.

The relationship between electrocution-related mortality and crime cannot be ignored. Stealing electricity and copper cables from major utility providers threatens not only the safety of the alleged thief, but an entire community. ${ }^{[12]} \mathrm{A}$ thorough evaluation of the scene of death may suggest a history of cable theft, since these deaths are usually unwitnessed. Secondary injuries suggesting a fall from a height are also quite common in these cases. ${ }^{[13]}$ Similar to the findings of Blumenthal ${ }^{[6]}$ in Gauteng, the majority of the cable theft cases occurred at night.

Some features describing the pattern of injuries (i.e. limb involvement, primary injuries and associated injuries) resembled the results of other studies reported in the literature. Unfortunately, the results pertaining specifically to the pathology of electrical burn wounds were inconclusive. Inconsistency in the manner of describing burn wounds, both macroscopically and microscopically, is presumably a reason why specific features associated with electrical burn wounds could not be identified in this study. As suggested by Blumenthal ${ }^{[6]}$ there is a need to develop a uniform way of medicolegal reporting.

\section{Conclusions}

Electrocution-related mortality is a common topic of discussion in the international literature, yet there is a paucity of data and research being conducted on fatal electrocutions in SA. Death from electric shock is preventable, and the importance of safety measures therefore cannot be ignored.

Although the postmortem findings in this study were nonspecific, the pathological profile of electrocution deaths was in keeping with descriptions of electrical burn wounds in the international literature. However, discrepancies were found in the reporting of these electrical burn wounds both in this study and in Blumenthal's study ${ }^{[6]}$ in Gauteng. A standardised system of reporting is therefore suggested. Various grading systems for the depth and extent of burn wounds already exist in clinical medicine. A suggestion would be to implement these existing classifications in the field of forensic medicine as well. Since the issue of reporting was noted in both SA studies, further research could perhaps focus on whether such an intervention will be beneficial in the reporting of burn wounds in postmortem examinations.

The need for better strategies to prevent electrocution has been emphasised worldwide. Although electrocution-related mortality is not a leading cause of death in the aforementioned high-prevalence areas, awareness should definitely be raised, especially focusing on the younger population.

Declaration. This research was done by SvC as part of an $\mathrm{MB} \mathrm{ChB}$ VI student intern research assignment in 2015.

Acknowledgements. None.

Author contributions. SvC conceived the idea, reviewed the literature, did data collection and wrote the manuscript; $\mathrm{CIH}$ and SAW reviewed the manuscript. Funding. None.

Conflicts of interest. None.

1. Gupta BD, Mehta RA, Trangadia MM. Profile of deaths due to electrocution: A retrospective study. J Indian Acad Forensic Med 2012;34(1):13-15

2. Sheikhazadi A, Kiani M, Ghadyani MH. Electrocution-related mortality: A survey of 295 deaths in Tehran, Iran between 2002 and 2006. Am J Forensic Med Pathol 2010;31(1):42-45. https://doi. org/10.1097/PAF.0b013e3181c213f6

3. Akcan R, Karacaoğlu E, Keten A, et al. Electrical fatalities in Ankara over 11 years. Turk J Med Sci 2012;42(3):533-538. https://doi.org/10.3906/sag-1010-1243

4. Lindström R, Byland P, Eriksson A. Accidental deaths caused by electricity in Sweden 1975 - 2000. J Forensic Sci 2006;55(6):1383-1888. https://doi.org/10.1111/j.1556-4029.2006.00257.x

5. Rautji R, Rudra A, Behra C, Dogra TD. Electrocution in South Delhi: A retrospective study. Med Sci Law 2003;43(4):350-352. https://doi.org/10.1258/rsmmsl.43.4.350

6. Blumenthal R. A retrospective descriptive study of electrocution deaths in Gauteng, South Africa: 2001 - 2004. Burns 2009;35(6):888-894. https://doi.org/10.1016/j.burns.2009.01.009

7. Statistics South Africa. Census of Western Cape Province, City of Cape Town Municipality, Statistics South Africa. Census of Western Cape Province, City of Cape Town Munis
Khayelitsha. 2011. http://www.statssa.gov.za/?page_id=4286\&id=328 (accessed 1 March 2018).

8. Tiemensma M, Burger EH. Sudden and unexpected deaths in an adult population, Cape Town, South Africa, 2001 - 2005. S Afr Med J 2012;102(2):90-94

9. Knight B, Saukko P. Knights' Forensic Pathology. 3rd ed. London: Edward Arnold, 2004:333.

10. Tirasci Y, Goren S, Subasi M, Grukan F. Electrocution related mortality: A review of 123 deaths in Diyarbakir, Turkey between 1996 and 2002. Tohoku J Exp Med 2006;208(2):141-145.

11. Bailey B, Forget S, Gaudreaulta P. Prevalence of potential risk factors in victims of electrocution. Forensic Sci Int 2001;123(1):58-62. https://doi.org/10.1016/S0379-0738(01)00525-4

12. Pretorius WL. A criminological analysis of copper cable theft in Gauteng. MA (Criminology) thesis. Pretoria: University of South Africa, December 2012.

13. Taylor AJ, McGwin G Jr, Brissie RM, et al. Death during theft from electricity utilities. Am J Forensic Med Pathol 2003;24(2):173-176. https://doi.org/10.1097/01.PAF.0000069581.13463.f1

Accepted 28 May 2018. 